OPEN ACCESS

Edited by:

Mårten Risling

Karolinska Institutet (KI), Sweden

Reviewed by:

Katherine N. Theken,

University of Pennsylvania,

United States

*Correspondence:

Slavica Kvolik

skvolik@mefos.hr

Sonja Skiljic

skiljicsonja@gmail.com

Specialty section:

This article was submitted to Neurotrauma,

a section of the journal

Frontiers in Neurology

Received: 21 November 2021 Accepted: 23 December 2021

Published: 25 January 2022

Citation:

Kvolik S, Koruga N and Skiljic S (2022) Analgesia in the Neurosurgical Intensive Care Unit.

Front. Neurol. 12:819613. doi: 10.3389/fneur.2021.819613

\section{Analgesia in the Neurosurgical Intensive Care Unit}

\author{
Slavica Kvolik ${ }^{1,2 *}$, Nenad Koruga ${ }^{1,3}$ and Sonja Skiljic ${ }^{1,2 *}$ \\ ${ }^{1}$ Faculty of Medicine, Josip Juraj Strossmayer University of Osijek, Osijek, Croatia, ${ }^{2}$ Department of Anesthesiology and \\ Critical Care, Osijek University Hospital, Osijek, Croatia, ${ }^{3}$ Department of Neurosurgery, Osijek University Hospital, Osijek, \\ Croatia
}

Acute pain in neurosurgical patients is an important issue. Opioids are the most used for pain treatment in the neurosurgical ICU. Potential side effects of opioid use such as oversedation, respiratory depression, hypercapnia, worsening intracranial pressure, nausea, and vomiting may be problems and could interfere with neurologic assessment. Consequently, reducing opioids and use of non-opioid analgesics and adjuvants ( $\mathrm{N}$-methyl-D-aspartate antagonists, $\alpha 2$-adrenergic agonists, anticonvulsants, corticosteroids), as well as non-pharmacological therapies were introduced as a part of a multimodal regimen. Local and regional anesthesia is effective in opioid reduction during the early postoperative period. Among non-opioid agents, acetaminophen and non-steroidal anti-inflammatory drugs are used frequently. Adverse events associated with opioid use in neurosurgical patients are discussed. Larger controlled studies are needed to find optimal pain management tailored to neurologically impaired neurosurgical patients.

Keywords: analgesia, pain, intensive care units, neurosurgery, opioids, drug side effects, constipation, gastroparesis

\section{INTRODUCTION}

Acute pain following acute brain injury could be substantial, but commonly it is not the primary consideration of the neurosurgical intensive care (NSICU) patients. The main reason for this is a priority of preventing secondary brain damage after trauma or surgical procedure $(1,2)$. Preservation of adequate cerebral perfusion and oxygenation while managing systemic intracranial pressure, mechanical ventilation, stabilization of circulation, fluid balance, temperature, and glycemic control in neurosurgical patients is complex and demanding but adequate pain control could improve outcome and patient satisfaction (3).

Several ERAS protocols (enhanced recovery after surgery) were recently developed for postoperative patients and perioperative pain treatment is included in them (4). Reduced preoperative fasting, maltodextrin fructose solution $2 \mathrm{~h}$ preoperatively, local infiltration of the surgical incision, and non-opioid analgesia are some of the maneuvers that were proven as efficient in awake patients (5). In addition to faster mobilization, these procedures also aim to reduce the use of opioids in the perioperative period, the side effect of which may be chronic opioid use and opioid dependence (6). It is usually easy to assess pain and administer appropriate pain medications in conscious patients, but it could be challenging in sedated, drowsy, or non-cooperative patients (7).

In this review, we will present the pain assessment and the most common modalities of analgesia in NSICU patients. We will discuss the problems associated with analgesia in patients with impaired consciousness, and complications of opioid use in patients with acute brain injury. Respiratory depression, gastrointestinal dysmotility, delirium, and addiction with possibilities to reduce them will be highlighted. 


\section{MULTIMODAL ANALGESIA POST-CRANIOTOMY}

Acute pain after craniotomy is considered moderate to severe during the first two postoperative days. It is often underrated and hard to estimate, potentially becoming chronic $(8,9)$. No analgesic regimen for post-craniotomy pain was proven as efficient for all patients $(1,10)$, although opioids provided superior acute pain relief compared to other drugs in small clinical studies (11). Despite their efficiency in pain control, potential side effects such as (over) sedation, respiratory depression, hypercapnia with worsening intracranial pressure, nausea, and vomiting may be problematic in neurosurgical patients and can interfere with neurologic assessment (12). The risk of opioid dependence limits safe opioid use for only a few days.

The introduction of the ERAS protocol in neurosurgery aims to accelerate postoperative recovery and length of stay in the ICU, and analgesia with fewer opioids in the early post-craniotomy period is crucial in achieving these goals. By adhering to these protocols, Elayat and co-workers reduced the length of stay in the ICU, reduced the number of episodes with VAS $>4$, and reduced opioid consumption in the patients undergoing elective craniotomy for supratentorial neurosurgery (5). Consequently, non-opioid analgesics as a part of a multimodal regimen with fewer side effects became standard of care in the postoperative pain treatment in the NSICU (3).

Local and regional anesthesia techniques, such as local scalp infiltration/block seem to be effective in the early postoperative period (13). Postoperative scalp infiltration was associated with a significant reduction in pain scores and the reduction of the opioid requirement over the $24 \mathrm{~h}$ postoperatively. No adverse events of the regional techniques were reported in a meta-analysis of the 7 RCTs with a total of 320 patients (13).

Among non-opioid agents, acetaminophen and nonsteroidal anti-inflammatory drugs are used most frequently in the first postoperative days to reduce the dose of opioids $(11,14)$. Recently various adjuvants were added as a part of preemptive, intraoperative and early postoperative analgesia for craniotomy pain. These include non-steroidal analgesics: $\mathrm{N}$-methyl-D-aspartate (NMDA) antagonists (ketamine), $\alpha 2$ -adrenergic agonists (dexmedetomidine) (10, 15, 16), as well as anticonvulsants (gabapentin and pregabalin) (17), corticosteroids, an intravenous local anesthetic (lidocaine), and non-pharmacological interventions, such as multipoint electro-acupuncture $(3,10,11)$.

Analgesia in NSICU patients with acute brain injury must not be a simple extension of post-craniotomy analgesia.

\section{PAIN ASSESSMENT TOOLS AND PATIENTS WITH ALTERED MENTAL STATUS}

Pain treatment in patients who have altered mental status before and after craniotomy is challenging. In NSICU patients, pain may be postoperative, e.g., after a craniotomy. It can be caused by intubation, mechanical ventilation, insufficient mobilization, as well as placement of nasogastric tube, urinary catheter, or central venous catheter. Several factors including sepsis, use of vasopressors, multiple comorbidities, previous (18), or new neurological deficiencies may influence pain assessment and treatment (19).

There are several tools adopted for the pain assessment in mechanically ventilated ICU patients, and patients with altered mental status. Behavioral Pain Scale (BPS), Critical Care Pain Observation Tool (CPOT) (20), or Nociception Coma ScaleRevised (NCS-R) (21) are some of the tools widely used. The parameters evaluated by these tools are physiologic parameters, verbal response, motor response, or facial mimic, which may be irreversibly impaired by brain damage. A study conducted by Nazari and coworkers showed that most of the behaviors that have been observed during painful stimulation in patients with traumatic brain injury included facial expressions, sudden eyeopening, frowning, lip changes, clear movements of extremities, neck stiffness, and sighing or moaning (22). Using these tools may be helpful, but also misleading in patients with brain edema after hypoxic brain injury, or in patients with intracranial hemorrhage and hypertension.

Severgnini et coworkers compared CPOT and BPS to examine their applicability in awake and sedated patients. They confirmed that both scales correlate with pain. In the comparison, CPOT was more sensitive (BPS 62.7\%, CPOT 76.5\%), and BPS was found to be more specific (BPS 91.7\%, CPOT 70.8\%) for pain assessment. The best results were obtained by combining both scales (23). In another study, Ribeiro and coworkers have studied psychometric properties of the BPS in traumatic brain injury. They found that BPS had good internal consistency, good discriminant validity, moderate to excellent reliability, and high responsiveness, but also that deep sedation affected the increase of grading during painful procedures (24).

Currently, none of the above rating scales can be considered the gold standard for pain assessment in neurocritical patients. As with other ICU patients, pain should be routinely assessed using validated behavioral scales and documented, especially before invasive procedures and physical therapy (24).

Implementation of standardized pain assessment protocols for the NSICU patients is important, but the actual implementation and adherence to these protocols remains an area for improvement and further investigations. Vital signs should not be used as a sole measurement, and surrogates for pain assessment (24). Using these tools may be helpful and misleading in patients with brain edema after hypoxic brain injury, or in patients with intracranial hemorrhage and hypertension.

The implementation of clinical assessment tools may lead to reduced use of analgesic and sedative agents, as observed in the nurses' study carried by Gelinas and coworkers (25). In the study of Phillips and coworkers, the implementation of the CPOT led to increased frequency of the pain assessment and a significant increase in the administration of paracetamol, opiates, propofol, patient-controlled analgesia, modified-release opiates, and neuropathic pain agents (26). A similar observation that implementation of pain assessment tools led to the more liberal administration of opioid-based pain relief was observed in Mascarenhas' study. Nurses in their study have increased opioid 
administration by $100 \%$ (27). The administration of opioids may decrease the pain experience, but may increase respiratory depression, have an impact on alertness and vigilance, and may impair neuropsychological evaluation.

The situation is more complicated when patients' condition dictates prolonged sedation, mechanical ventilation, and when other invasive and painful procedures are routinely performed in NSICU. In such situations, a protocol called "analgesia first sedation" may be useful by increasing compliance with the ventilator but may lead to increased opioid use (27). In neurologically impaired patients, it may mask their neurologic assessment.

\section{ACUTE BRAIN INJURY AND PAIN CONTROL}

In patients with acute brain injury, intracranial pressure oscillations are associated with hemodynamic instability and are treated with a reduction in intracranial pressure by continuous use of sedatives, such as propofol and opiates. Sedatives and opioids are given commonly, based on the blood pressure and pulse values to maintain smooth circulation and mechanical ventilation (28). Different opioids may be used like fentanyl, sufentanil, remifentanil, and morphine. Opioids are beneficial for analgesia, but their bolus administration may increase ICP with associated decreases in MAP and CPP, and should be avoided (29). In the systematic review Wiener and coworkers have found no consistent results between different opioids, and between opioid and non-opioid analgesia in the management of traumatic brain injury regarding their effects on MAP, ICP, or CPP (29). Opioid consumption can be reduced by the use of dexmedetomidine for sedation without affecting neurological function (30).

\section{Paroxysmal Sympathetic Hyperactivity}

Paroxysmal sympathetic hyperactivity (PSH) is a syndrome resulting usually from traumatic brain injury $(79.4 \%$ of the patients), and rarely from hypoxia $(9.7 \%)$ or cerebrovascular accident (5.4\%) (31). Since these conditions are commonly accompanied by severe brain swelling or blood extravasation, pain could be very strong although it is rarely reported and/or observed. PSH is characterized by simultaneous, paroxysmal transient increases in sympathetic (elevated heart rate, blood pressure, respiratory rate, temperature, sweating) and motor activity (31). Analgesia is particularly demanding in patients with impaired mental status and in those who have developed PSH (18). In patients with PSH, these symptoms may resemble withdrawal syndrome or acute pain (Table 1) and are very often treated with opioids (32-34). Until now, there are no studies reporting pain scores using any of the pain assessment tools in $\mathrm{PSH}$ patients.

Common problems with $\mathrm{PSH}$ are difficult mechanical ventilation, difficult maintenance of fluid and electrolyte balance, management of increased body temperature, increased number of blood specimens, and unresponsiveness of the symptoms to treatment.
TABLE 1 | The most common symptoms of paroxysmal sympathetic hyperactivity, withdrawal syndrome, and acute pain in neurosurgical patients.

\begin{tabular}{llll}
\hline & $\begin{array}{l}\text { Paroxysmal } \\
\text { sympathetic } \\
\text { hyperactivity }\end{array}$ & $\begin{array}{l}\text { Opioid } \\
\text { withdrawal } \\
\text { syndrome }\end{array}$ & Acute pain \\
\hline Tachycardia & ++ & ++ & ++ \\
Hypertension & ++ & ++ & ++ \\
Hyperventilation & ++ & + & + \\
Fever & ++ & + (or & - \\
& & hypothermia) & \\
Profuse sweating & ++ & + & ++ \\
Restlesness & + & ++ & + \\
Muscle rigidity and hypertonus & ++ & Movement & + /- \\
& & disorders, & \\
& & tremor & \\
Agitation, insomnia & & ++ & + \\
Other symptoms & & Rhinorrhoea, & $\uparrow$ ICP \\
& Typical posturing & lacrimation, & \\
& & nausea, & \\
& & vomiting, & \\
& & diarrhea & \\
& & &
\end{tabular}

Note: ++ present, + sometimes present, +/- not always present, - not registered, $\uparrow$ increase.

One of the treatment goals in brain-injured patients is symptoms prevention and reduction (15). The symptoms of $\mathrm{PSH}$ are usually treated with various drugs, including morphine, non-selective $\beta$-blockers, short-acting benzodiazepines, baclofen, clonidine, non-steroidal anti-inflammatory drugs, $\alpha 2$ agonists, and GABA agonists. Although opioids and benzodiazepines are capable to control breakthrough episodes, due to their sedative effects and the possibility of addiction, other drug classes are gaining more popularity (35).

Currently, dexmedetomidine and gabapentin are used for both symptom and opioid reduction. In a recent study, Peng and coworkers have confirmed that dexmedetomidine reduces paroxysmal hypertension, average time for normalization of body temperature, heart rate, and respiratory rate below 25 breaths per minute, but does not protect against the recurrence of PSH (36). In the patients with severe traumatic brain injury and symptoms of paroxysmal sympathetic hyperactivity who underwent surgery, dexmedetomidine was not capable of either reducing ICU and hospital stay or of improving Glasgow outcome score (37).

Gabapentin is a promising drug for the treatment of PSH. Godo and colleagues reported a case series of patients who were resistant to benzodiazepines, opioids, and non-steroidal antiinflammatory drugs. Initial gabapentin doses of $200 \mathrm{mg} 3$ times a day increased to $400 \mathrm{mg} 3$ times a day resulted in a reduction of PSH symptoms (32). To achieve wider use of gabapentin for PSH treatment, its effects observed in the single institution must be confirmed in a larger placebo-controlled study.

\section{ADVERSE EVENTS OF OPIOIDS IN NEUROSURGICAL PATIENTS}

Although some preclinical studies have suggested that opioid receptor agonists may be beneficial in brain injury, capable of 
reducing brain edema as well as of providing neuroprotection during a stroke (38), these preclinical observations were not confirmed in clinical studies. Moreover, the use of opioids as an analgesic regimen in neurosurgical patients is associated with several clinical problems (side effects) such as oversedation, respiratory depression, prolonged mechanical ventilation, truncal rigidity, inappropriate immune modulation, development of opioid tolerance, opioid-induced hyperalgesia, ICU addiction, and delirium (39). Opioids also have direct cardiovascular effects, decreasing blood pressure, causing vasodilation, and decreasing cardiac work (40). Gastrointestinalrelated side effects, which include constipation, nausea, vomiting, dry mouth, gastro-esophageal reflux, abdominal cramping, spasms, and bloating, are well-known as opioid-induced bowel dysfunction. Opioid-induced constipation (OIC) in patients receiving opioids is persistent and the most frequently reported side effect $(41,42)$.

\section{Respiratory Depression}

Respiratory depression with consequent hypoxia and permanent brain injury has often been described after acute opioid intoxication (43). The outcomes of these patients are associated with the duration and severity of hypoxia. There are fewer studies correlating outcomes of the patients with acute brain trauma regarding opioid use and the duration of mechanical ventilation. Dexmedetomidine and propofol allow patients to wake up faster and breathe easier, although they are usually used with opioids in patients with acute brain injury. In the patients with acute brain injury, both dexmedetomidine and dexmedetomidine with propofol were associated with a significantly higher rate of hypotension as compared to propofol only or no sedation (44).

\section{Gastroparesis and Constipation}

Gastrointestinal dysmotility, i.e., gastroparesis and bowel paralysis are commonly observed in the acute phase of injury in neurosurgical patients $(45,46)$. Both disorders are a complication of opioid use and brainstem lesions (Table 2). In 139 mechanically ventilated patients in general ICU, the incidence of opioid-induced constipation (OIC) was 63\%, and gastric retention was $49 \%$ (53). Guerra and coworkers found OIC in $72 \%$ of critically ill patients (54). In their paper, by the study protocol patients on the parenteral nutrition therapy were excluded. The incidence of constipation would probably be even higher if such patients were accounted.

Kieninger and coworkers studied a promotility protocol in adult NSICU patients with acute severe brain injury and anticipated mechanical ventilation for more than 3 days (55). All patients received sufentanil and sedation. Promotility procedures were colon massage, physical therapy, and early peroral lactulose, followed by naloxone $4 \mathrm{mg}$ p.o. $3 \mathrm{x} /$ day. In the study group, an adequate defecation pattern was observed in 9 out of 37 (24.3\%) compared to only 9 out of $109(8.3 \%)$ in the control group (55). Opioid antagonists are considered a logical approach to treat OIC. In a recent study, both enteral naloxone and subcutaneous methylnaltrexone were effective for the treatment of OIC in medical ICU. The median times to first bowel movement were 30 and $24 \mathrm{~h}$ for naloxone and methylnaltrexone patients, respectively (56).

Schmittner and coworkers reported no difference in the time period until full enteral nutrition or first defecation between patients receiving opioid fentanyl and $\mathrm{S}(+)$-ketamine in neurosurgical patients (57). This suggests that brain damage, per se, may influence gut dysfunction via unknown mechanisms. Larger prospective studies are needed to answer the true OIC incidence and optimal treatment in NSICU patients with brain injury.

\section{Hypotension}

A decrease in the mean arterial blood pressure is wellknown during fentanyl, remifentanil, or alfentanil administration (58), as well as during prolonged sedation in mechanically ventilated patients and it is accompanied by increased use of vasopressors in the medical ICU (59). The total amount of propofol and fentanyl correlated with vasopressor use and prolonged sedation. Patients who underwent analgesia with $S(+)$-ketamine showed a trend to lower demand for norepinephrine compared with the fentanyl group without an increase of ICP and CPP (57).

\section{Addiction in the ICU}

Addicted patients are a growing category in ICU. Analyzing toxicological samples taken from 44 patients admitted to the Intensive Care Unit Ruiz-García and coworkers confirmed the use of $\geq 1$ substance in $74 \%$ of patients. The most consumed substances were alcohol and tobacco ( $>55 \%$ ), and cannabis, amphetamines (> 11\%), and cocaine (9\%). Discontinuation of each of these substances can lead to withdrawal syndrome (60).

Opioid addiction is reflected in an individual pathologically pursuing reward and/or relief by substance use and other behaviors. Usually, they need a special approach while on opioid maintenance treatment during the ICU stay, since the opioid restriction is not recommended during the acute illness (61). The toxic effect of opioids and other substances of abuse may lead to ischemic brain damage and ICU admission in youth and younger adults (62). Non-traumatic hypoxic brain injury was observed in a total of $8 \%$ of the patients admitted into the ICU with opioid overdose with mortality of $10 \%$ (63). In the period after hypoxic brain injury, during the recovery in ICU, these patients may develop symptoms of withdrawal syndrome (Table 1) (64).

Withdrawal syndrome may be a serious complication during ICU treatment (33). The most severe symptoms can be experienced in patients who have discontinued opioid therapy at the NSICU, regardless of whether the patient was taking opioids before admission to the NSICU, or opioid therapy as part of analgesia during NSICU treatment. Risk factors associated with iatrogenic withdrawal syndrome include duration of therapy and cumulative drug dose (65). Critically ill patients with preexisting cognitive or functional 
TABLE 2 | Frequency of the most common gastrointestinal motility disorders in the neurosurgical intensive care unit and their association with risk factors.

\begin{tabular}{|c|c|c|c|}
\hline References & Postinjury phase & GI motility disorder (\%) & Risk factors \\
\hline Lim et al. (47) & Acute poststroke & Constipation 39\% & $\begin{array}{l}\text { Immobility, bedpan use, a longer length of } \\
\text { stay }\end{array}$ \\
\hline Vieira et al. (48) & $\begin{array}{l}\text { Traumatic brain injury- acute } \\
\text { postinjury phase }\end{array}$ & Diarrhea 69.6\% & $\begin{array}{l}\text { Critical illness, enteral nutrition, antibiotics } \\
\text { usage }\end{array}$ \\
\hline Makkar et al. (49) & $\begin{array}{l}\text { Traumatic brain injury- acute } \\
\text { postinjury phase }\end{array}$ & $\begin{array}{l}\text { Gastroparesis, gastric aspirate volume } \\
\text { (GAV) } 60.5 \%\end{array}$ & $\begin{array}{l}\text { Raised intracranial pressure, sympathetic } \\
\text { stimulation, hyperglycemia, use of opioids. }\end{array}$ \\
\hline Pinto et al. (45) & $\begin{array}{l}\text { Traumatic brain injury- acute } \\
\text { postinjury phase }\end{array}$ & Feeding intolerance $75.0 \%$ & Brain-gut dysfunction \\
\hline Berry et al. (50) & $\begin{array}{l}\text { Traumatic brain injury- acute } \\
\text { postinjury phase }\end{array}$ & $\begin{array}{l}\text { No bowel movement between } 48 \text { and } \\
72 \mathrm{~h} 45.6 \%\end{array}$ & $\begin{array}{l}\text { Autonomic disturbances, hyper-sympathetic } \\
\text { response, damage of hypothalamus, narcotic } \\
\text { analgesics }\end{array}$ \\
\hline Cai et al. (51) & Acute recovery sequelae phase & $\begin{array}{l}\text { Constipation } 41.6 \% \text { Constipation } 31.5 \\
\% \text { Constipation } 22.6 \%\end{array}$ & $\begin{array}{l}\text { Incidence higher with acute phase, basal } \\
\text { ganglia inclusion, and cerebral hemorrhage }\end{array}$ \\
\hline Robain et al. (52) & $\begin{array}{l}\text { Rehabilitation after recent vascular } \\
\text { hemiplegia }\end{array}$ & Constipation 60\% & $\begin{array}{l}\text { Brain lesions, functional status of patients } \\
\text { (assessed by Barthel Index) }\end{array}$ \\
\hline Cheng et al. (46) & Chronic & Constipation & $\begin{array}{l}\text { Brainstem lesions, the desire for defecation } \\
\text { threshold, physical activity level }\end{array}$ \\
\hline
\end{tabular}

impairment are at special risk to develop opioid withdrawal syndrome (66).

In NSICU where opioids are commonly used, withdrawal may be difficult to recognize. Its recognition could be even poorer because no valid tools were developed for ICU patients. Opioid withdrawal syndrome may mimic PSH or other manifestations of brain damage (Table 1). Opioid doses should be kept at a minimum, and non-opioid drugs are preferred, whenever their use is effective. There are several strategies for opioid weaning, including scheduled enteral opioids after continuous opioid infusions (66) and the use of non-opioid drugs like dexmedetomidine and clonidine.

Promoting non-opioid analgesics and reducing opioid use is important to minimize the use of opioids in previously opioidnaïve patients after discharge from the NSICU. To date, there are no studies to confirm the frequency of opioid use in neurosurgical patients after discharge from the ICU. Data from other fields of intensive care confirm that this proportion is significant. Based on the recent cohort studies $40.8 \%$ (346 in 848) medical ICU patients and $45 \%$ (32 of the 71 surgical) opiate-naïve patients were discharged with a new opioid prescription $(67,68)$. A shorter inpatient opioid therapy decreased the risk of postdischarge opioid therapy (68).

In clinical situations where opioid use cannot be avoided, nor can opioids be replaced by other drugs, opioid rotation may be useful. It can reduce both risks of opioid dependence and of opioid tolerance, i.e., increase the opioid dose to maintain equianalgesic effects $(39,69)$.

\section{ICU Delirium}

Patients may develop delirium as a consequence of opioid administration in the ICU. Pisani and coworkers reported patients who received opioids had a longer duration of delirium (70). The incidence of delirium in neurosurgical patients may be as high as $42.2 \%$ as observed by Wang and coworkers (71), but a correlation between delirium and opioid use was not analyzed in the study. Opioid use in NSICU is a modifiable risk factor for delirium.

\section{UNANSWERED QUESTIONS IN THE FIELD}

There are numerous unresolved questions in the field of analgesic use in acute brain injury, such as whether the choice of analgesics may influence neurological recovery in acute brain injury. In the in vivo study tramadol was able to minimize perivascular edema, neuronal necrosis, inflammatory cell infiltration in acute and chronic ischemia/reperfusion injury (72). The comparable neuroprotective effect was observed with dexmedetomidine in in vitro and in vivo studies. Possible mechanisms are signaling pathways for inflammatory response, oxidative stress, neurotransmitter regulation, mitochondrial function, apoptotic pathway, and autophagy (73). This effect was not confirmed in human studies. It is also unknown whether opioid rotation can reduce the incidence of ICU withdrawal syndrome, and postdischarge opioid use. It has been confirmed that the use of multimodal treatment under the ERAS protocol, such as the combination of non-opioid analgesics, and gabapentin, reduces opioid use in postoperative general surgical patients (74). While decreasing pain scores, it decreased the level of consciousness in a dose-dependent fashion, and prolonged stay in the postanesthesia care unit, too (74). There are no such studies in the patients who suffered an acute brain injury. It is particularly unknown whether the neurological outcome may be modified in the patients who have received such a multimodal treatment. This should be confirmed by future studies.

\section{CONCLUSION}

Both pain assessment and pain control are challenging in neurosurgical patients with altered consciousness. Opioid use is common in NSICU but can lead to respiratory depression 
and difficult neurological evaluation from oversedation. Avoiding opioids and the use of alternative medications and therapies are recommended. Multimodal postoperative analgesia and proper drug selection in the ICU may reduce the side effects of opioid treatment. Further studies should confirm whether the choice of analgesia and opioid restriction in patients with severe brain injury may influence outcomes.

\section{AUTHOR CONTRIBUTIONS}

SS and SK each wrote sections of the paper and performed the literature review. SK and NK have prepared tables for publication. SK, NK, and SS have approved a final version of the

\section{REFERENCES}

1. Bösel J. Moderne prinzipien der neurologischen intensivmedizin. Nervenarzt. (2018) 89:1411-27. doi: 10.1007/s00115-018-0634-x

2. Borsook D, Upadhyay J, Chudler EH, Becerra L. A key role of the basal ganglia in pain and analgesia-insights gained through human functional imaging. $\mathrm{Mol}$ Pain. (2010) 6:27. doi: 10.1186/1744-8069-6-27

3. Morad A, Farrokh S, Papangelou A. Pain management in neurocritical care; an update. Curr Opin Crit Care. (2018) 24:72-79. doi: 10.1097/MCC.0000000000000480

4. Ali ZS, Ma TS, Ozturk AK, Malhotra NR, Schuster JM, Marcotte PJ, et al. Pre-optimization of spinal surgery patients: development of a neurosurgical enhanced recovery after surgery (ERAS) protocol. Clin Neurol Neurosurg. (2018) 164:142-53. doi: 10.1016/j.clineuro.2017.12.003

5. Elayat A, Jena SS, Nayak S, Sahu RN, Tripathy S. Enhanced recovery after surgery - ERAS in elective craniotomies-a non-randomized controlled trial. BMC Neurol. (2021) 21:127. doi: 10.1186/s12883-021-02150-7

6. Seal KH, Bertenthal D, Barnes DE, Byers AL, Gibson CJ, Rife TL, et al. Traumatic brain injury and receipt of prescription opioid therapy for chronic pain in iraq and afghanistan veterans: do clinical practice guidelines matter? $J$ Pain. (2018) 19:931-41. doi: 10.1016/j.jpain.2018.03.005

7. Maier J, Fröhlich MR, Spirig R. [Standardized impartial assessment of pain in unconscious patients with the Zurich observation pain assessment on a neurosurgical intensive care unit - a case report]. Pflege. (2015) 28:1931. doi: 10.1024/1012-5302/a000401

8. Lutman B, Bloom J, Nussenblatt B, Romo V. A contemporary perspective on the management of post-craniotomy headache and pain. Curr Pain Headache Rep. (2018) 22:69. doi: 10.1007/s11916-018-0722-4

9. Grodofsky S. Chronic pain in neurosurgery. Anesthesiol Clin. (2016) 34:47995. doi: 10.1016/j.anclin.2016.04.003

10. Tsaousi GG, Logan SW, Bilotta F. Postoperative pain control following craniotomy: a systematic review of recent clinical literature. Pain Pract. (2017) 17:968-81. doi: 10.1111/papr.12548

11. Zeiler FA, AlSubaie F, Zeiler K, Bernard F, Skrobik Y. Analgesia in neurocritical care: an international survey and practice audit*. Crit Care Med. (2016) 44:973-80. doi: 10.1097/CCM.0000000000001602

12. Dunn LK, Naik BI, Nemergut EC, Durieux ME. Post-Craniotomy pain management: beyond opioids. Curr Neurol Neurosci Rep. (2016) 16:93. doi: 10.1007/s11910-016-0693-y

13. Guilfoyle MR, Helmy A, Duane D, Hutchinson PJA. Regional scalp block for postcraniotomy analgesia: a systematic review and metaanalysis. Anesth Analg. (2013) 116:1093-102. doi: 10.1213/ANE.0b013e31828 $63 \mathrm{c} 22$

14. Puntis M, Garner A. Management of pain following craniotomy. Br J Nurs. (2015) 24:740-4. doi: 10.12968/bjon.2015.24.14.740

15. Peng K, Jin X, Liu S, Ji F. Effect of intraoperative dexmedetomidine on post-craniotomy pain. Clin Ther. (2015) 37:1114-21.e1. doi: 10.1016/j.clinthera.2015.02.011 manuscript. All authors contributed to the article and approved the submitted version.

\section{FUNDING}

This manuscript was supported by IP16-20 and IP1-21 by J.J. Strossmayer University, Medical Faculty, Osijek, Croatia.

\section{ACKNOWLEDGMENTS}

The authors acknowledge Branko Bulovic (Library, University of New South Wales, Sydney, Australia) for help with language editing.

16. Vallapu S, Panda N, Samagh N, Bharti N. Efficacy of dexmedetomidine as an adjuvant to local anesthetic agent in scalp block and scalp infiltration to control postcraniotomy pain: a double-blind randomized trial. J Neurosci Rural Pract. (2018) 9:73-9. doi: 10.4103/jnrp.jnrp_310_17

17. Shay JE, Kattail D, Morad A, Yaster M. The postoperative management of pain from intracranial surgery in pediatric neurosurgical patients. Pediatr Anesth. (2014) 24:724-33. doi: 10.1111/pan.12444

18. Naro A, Bramanti P, Bramanti A, Calabrò RS. Assessing pain in patients with chronic disorders of consciousness: are we heading in the right direction? Conscious Cogn. (2017) 55:148-55. doi: 10.1016/j.concog.2017.08.009

19. Booth CM, Boone RH, Tomlinson G, Detsky AS. Is this patient dead, vegetative, or severely neurologically impaired?assessing outcome for comatose survivors of cardiac arrest. JAMA. (2004) 291:870-9. doi: 10.1001/jama.291.7.870

20. Chanques G, Pohlman A, Kress JP, Molinari N, de Jong A, Jaber S, et al. Psychometric comparison of three behavioural scales for the assessment of pain in critically ill patients unable to self-report. Crit Care. (2014) 18:R160. doi: 10.1186/cc14000

21. Chatelle C, Thibaut A, Whyte J, De Val MD, Laureys S, Schnakers C. Pain issues in disorders of consciousness. Brain Inj. (2014) 28:12028. doi: 10.3109/02699052.2014.920518

22. Nazari R, Pahlevan Sharif S, Allen KA, Sharif Nia H, Yee B-L, Yaghoobzadeh A. Behavioral pain indicators in patients with traumatic brain injury admitted to an intensive care unit. J Caring Sci. (2018) 7:197203. doi: $10.15171 /$ jcs. 2018.030

23. Severgnini P, Pelosi P, Contino E, Serafinelli E, Novario R, Chiaranda M. Accuracy of critical care pain observation tool and behavioral pain scale to assess pain in critically ill conscious and unconscious patients: prospective, observational study. J Inten Care. (2016) 4:68. doi: 10.1186/s40560-016-0192-x

24. Ribeiro CJN, Lima AGCF, de Araújo RAS, Nunes M da S, Alves JAB, Dantas DV, et al. Psychometric properties of the behavioral pain scale in traumatic brain injury. Pain Manag Nurs Off J Am Soc Pain Manag Nurses. (2019) 20:152-7. doi: 10.1016/j.pmn.2018.09.004

25. Gélinas C, Arbour C, Michaud C, Vaillant F, Desjardins S. Implementation of the critical-care pain observation tool on pain assessment/management nursing practices in an intensive care unit with nonverbal critically ill adults: a before and after study. Int J Nurs Stud. (2011) 48:1495504. doi: 10.1016/j.ijnurstu.2011.03.012

26. Phillips ML, Kuruvilla V, Bailey M. Implementation of the critical care pain observation tool increases the frequency of pain assessment for noncommunicative ICU patients. Aust Crit Care. (2019) 32:36772. doi: 10.1016/j.aucc.2018.08.007

27. Mascarenhas M, Beattie M, Roxburgh M, MacKintosh J, Clarke N, Srivastava D. Using the model for improvement to implement the critical-care pain observation tool in an adult intensive care unit. BMJ open Qual. (2018) 7:e000304. doi: 10.1136/bmjoq-2017-000304

28. Roberts DJ, Hall RI, Kramer AH, Robertson HL, Gallagher CN, Zygun DA. Sedation for critically ill adults with severe traumatic brain injury: a 
systematic review of randomized controlled trials. Crit Care Med. (2011) 39:2743-51. doi: 10.1097/CCM.0b013e318228236f

29. Wiener J, McIntyre A, Janzen S, Mirkowski M, MacKenzie HM, Teasell R. Opioids and cerebral physiology in the acute management of traumatic brain injury: a systematic review. Brain Inj. (2019) 33:559-66 doi: 10.1080/02699052.2019.1574328

30. Humble SS, Wilson LD, Leath TC, Marshall MD, Sun DZ, Pandharipande PP, et al. ICU sedation with dexmedetomidine after severe traumatic brain injury. Brain Inj. (2016) 30:1266-70. doi: 10.1080/02699052.2016.1187289

31. Perkes I, Baguley IJ, Nott MT, Menon DK. A review of paroxysmal sympathetic hyperactivity after acquired brain injury. Ann Neurol. (2010) 68:126-35. doi: 10.1002/ana.22066

32. Godo S, Irino S, Nakagawa A, Kawazoe Y, Fujita M, Kudo D, et al. Diagnosis and management of patients with paroxysmal sympathetic hyperactivity following acute brain injuries using a consensus-based diagnostic tool: a single institutional case series. Tohoku J Exp Med. (2017) 243:118. doi: $10.1620 /$ tjem.243.11

33. Taesotikul S, Dilokpattanamongkol P, Tangsujaritvijit V, Suthisisang C. Incidence and clinical manifestation of iatrogenic opioid withdrawal syndrome in mechanically ventilated patients. Curr Med Res Opin. (2021) 37:1213-9. doi: 10.1080/03007995.2021.1928616

34. Peng Y, Zhang W, Kass IS, Han R. Lidocaine reduces acute postoperative pain after supratentorial tumor surgery in the pacu: a secondary finding from a randomized, controlled trial. J Neurosurg Anesthesiol. (2016) 28:30915. doi: 10.1097/ANA.0000000000000230

35. Raithel DS, Ohler KH, Porto I, Bicknese AR, Kraus DM. Morphine: an effective abortive therapy for pediatric paroxysmal sympathetic hyperactivity after hypoxic brain injury. J Pediatr Pharmacol Ther. (2015) 20:33540. doi: 10.5863/1551-6776-20.4.335

36. Peng Y, Zhu H, Chen H, Zhu Z, Zhou H, Zhang S, et al. Dexmedetomidine attenuates acute paroxysmal sympathetic hyperactivity. Oncotarget. (2017) 8:69012-9. doi: 10.18632/oncotarget.16920

37. Tang Q, Wu X, Weng W, Li H, Feng J, Mao Q, et al. The preventive effect of dexmedetomidine on paroxysmal sympathetic hyperactivity in severe traumatic brain injury patients who have undergone surgery: a retrospective study. PeerJ. (2017) 5:e2986. doi: 10.7717/peerj.2986

38. Yang L, Wang H, Shah K, Karamyan VT, Abbruscato TJ. Opioid receptor agonists reduce brain edema in stroke. Brain Res. (2011) 1383:30716. doi: 10.1016/j.brainres.2011.01.083

39. Martyn JAJ, Mao J, Bittner EA. Opioid tolerance in critical illness. $N$ Engl J Med. (2019) 380:365-78. doi: 10.1056/NEJMra1800222

40. LiverTox: Clinical and Research Information on Drug-Induced Liver Injury. Bethesda, MD: National Institute of Diabetes and Digestive and Kidney Diseases (2012).

41. Müller-Lissner S, Bassotti G, Coffin B, Drewes AM, Breivik H, Eisenberg E, et al. Opioid-induced constipation and bowel dysfunction: a clinical guideline. Pain Med. (2017) 18:1837-63. doi: 10.1093/pm/pnw255

42. Streicher JM, Bilsky EJ. Peripherally acting $\mu$-opioid receptor antagonists for the treatment of opioid-related side effects: mechanism of action and clinical implications. J Pharm Pract. (2018) 31:658-69. doi: 10.1177/0897190017732263

43. Oladunjoye AO, Oladunjoye OO, Olubiyi O, Yee MR, Espiridion ED. Predictors and outcomes of invasive mechanical ventilation in opioid overdose hospitalization in the United States. Cureus. (2020) 12:e9788. doi: $10.7759 /$ cureus. 9788

44. Pajoumand M, Kufera JA, Bonds BW, Devabhakthuni S, Boswell S, Hesselton K, et al. Dexmedetomidine as an adjunct for sedation in patients with traumatic brain injury. J Trauma Acute Care Surg. (2016) 81:34551. doi: $10.1097 /$ TA. 0000000000001069

45. Pinto TF, Rocha R, Paula CA, de Jesus RP. Tolerance to enteral nutrition therapy in traumatic brain injury patients. Brain Inj. (2012) 26:11137. doi: 10.3109/02699052.2012.666369

46. Cheng J, Li L, Xu F, Xu Y, Lin L, Chen JDZ. Poststroke constipation is associated with impaired rectal sensation. Am J Gastroenterol. (2020) 115:10514. doi: 10.14309/ajg.0000000000000479

47. Lim S-F, Ong SY, Tan YL, Ng YS, Chan YH, Childs C. Incidence and predictors of new-onset constipation during acute hospitalisation after stroke. Int J Clin Pract. (2015) 69:422-8. doi: 10.1111/ijcp.12528
48. Vieira LV, Pedrosa LAC, Souza VS, Paula CA, Rocha R. Incidence of diarrhea and associated risk factors in patients with traumatic brain injury and enteral nutrition. Metab Brain Dis. (2018) 33:175560. doi: 10.1007/s11011-018-0287-2

49. Makkar JK, Gauli B, Jain K, Jain D, Batra YK. Comparison of erythromycin versus metoclopramide for gastric feeding intolerance in patients with traumatic brain injury: a randomized double-blind study. Saudi J Anaesth. (2016) 10:308-13. doi: 10.4103/1658-354X.174902

50. Berry JAD, Ogunlade J, Kashyap S, Berry DK, Wacker M, Miulli $\mathrm{DE}$, et al. Clinical efficacy of mesenteric lift to relieve constipation in traumatic brain injury patients. J Am Osteopath Assoc. (2020) 597600. doi: 10.7556/jaoa.2020.094

51. Cai W, Wang L, Guo L, Wang J, Zhang X, Cao W, et al. Correlation analysis between post-stroke constipation and brain injury. Nan Fang Yi Ke Da Xue Xиe Bao. (2013) 33:117-20. Chinese.

52. Robain G, Chennevelle JM, Petit F, Piera JB. Incidence de la constipation dans une population de patients atteints d'hémiplégie vasculaire récente: étude prospective de 152 cas [Incidence of constipation after recent vascular hemiplegia: a prospective cohort of 152 patients]. Rev Neurol (Paris). (2002) 158:589-92.

53. Pérez-Sánchez J, Fernández-Boronat J, Martínez-Méndez E, MarínCagigas ML, Mota-Puerto D, Pérez-Román MC, et al. Evaluation and handling of constipation in critical patients. Enferm Int. (2017) 28:160-8. doi: 10.1016/j.enfie.2017.10.003

54. Guerra TL de S, Mendonça SS, Marshall NG. Incidence of constipation in an intensive care unit. Rev Bras Ter intensiva. (2013) 25:87-92. doi: 10.5935/0103-507X.20130018

55. Kieninger M, Sinner B, Graf B, Grassold A, Bele S, Seemann M, et al. Standardized application of laxatives and physical measures in neurosurgical intensive care patients improves defecation pattern but is not associated with lower intracranial pressure. Crit Care Res Pract. (2014) 2014:367251. doi: 10.1155/2014/367251

56. Merchan C, Altshuler D, Papadopoulos J. Methylnaltrexone versus naloxone for opioid-induced constipation in the medical intensive care unit. Ann Pharmacother. (2017) 51:203-8. doi: 10.1177/1060028016677310

57. Schmittner MD, Vajkoczy SL, Horn P, Bertsch T, Quintel M, Vajkoczy P, et al. Effects of fentanyl and $s(+)$-ketamine on cerebral hemodynamics, gastrointestinal motility, and need of vasopressors in patients with intracranial pathologies: a pilot study. J Neurosurg Anesthesiol. (2007) 19:25762. doi: 10.1097/ANA.0b013e31811f3feb

58. Fodale V, Schifilliti D, Praticò C, Santamaria LB. Remifentanil and the brain. Acta Anaesthesiol Scand. (2008) 52:31926. doi: $10.1111 / j .1399-6576.2007 .01566 . x$

59. Mukhopadhyay A, Tai BC, Remani D, Phua J, Cove ME, Kowitlawakul Y. Age related inverse dose relation of sedatives and analgesics in the intensive care unit. PLoS ONE. (2017) 12:e0185212. doi: 10.1371/journal.pone.0185212

60. Ruiz-García T, Nogué-Xarau S, Zavala-Zegarra E, Cirera-Guasch A, RíosGuillermo J. Need for sedation and analgesia in patients with a history of substance misuse admitted to an intensive care unit. Nurs Crit Care. (2016) 21:358-66. doi: 10.1111/nicc. 12117

61. Vearrier L. The value of harm reduction for injection drug use: a clinical and public health ethics analysis. Disease Month. (2018) 65(5):11941. doi: 10.1016/j.disamonth.2018.12.002

62. Chan V, Pole JD, Keightley M, Mann RE, Colantonio A. Children and youth with non-traumatic brain injury: a population based perspective. BMC Neurol. (2016) 16:110. doi: 10.1186/s12883-016-0631-2

63. Stevens JP, Wall MJ, Novack L, Marshall J, Hsu DJ, Howell MD. The critical care crisis of opioid overdoses in the United States. Ann Am Thorac Soc. (2017) 14:1803-9. doi: 10.1513/AnnalsATS.201701-022OC

64. Hamlin DW, Hussain N, Pathare A. Storms and silence: a case report of catatonia and paroxysmal sympathetic hyperactivity following cerebral hypoxia. BMC Psychiatry. (2020) 20:473. doi: 10.1186/s12888-020-02878-5

65. Best KM, Boullata JI, Curley MAQ. Risk factors associated with iatrogenic opioid and benzodiazepine withdrawal in critically ill pediatric patients: a systematic review and conceptual model. Pediatr Crit Care Med. (2015) 16:175-83. doi: 10.1097/PCC.0000000000000306

66. Best KM, Wypij D, Asaro LA, Curley MAQ. Patient, process, and system predictors of iatrogenic withdrawal syndrome in critically ill 
children*. Crit Care Med. (2017) 45:e7-e15. doi: 10.1097/CCM.000000000000 1953

67. Tollinche LE, Seier KP, Yang G, Tan KS, Tayban YD, Pastores SM, et al. Discharge prescribing of enteral opioids in opioid naïve patients following non-surgical intensive care: a retrospective cohort study. J Crit Care. (2021) 68:16-21. doi: 10.1016/j.jcrc.2021.10.021

68. Academia EC, Gabriel CJ, Mueller A, Schwarz KA, Bartels K, Valuck RJ, et al. Opioid prescribing after discharge in a previously mechanically ventilated, opioid-naïve cohort. Ann Pharmacother. (2020) 54:1065-72. doi: 10.1177/1060028020919122

69. Sanavia E, Mencía S, Lafever SN, Solana MJ, Garcia M, López-Herce J. Sedative and analgesic drug rotation protocol in critically ill children with prolonged sedation: evaluation of implementation and efficacy to reduce withdrawal syndrome. Pediatr Crit care Med J Soc Crit Care Med World Fed Pediatr Int Crit Care Soc. (2019) 20:1111-7. doi: 10.1097/PCC.0000000000002071

70. Pisani MA, Murphy TE, Araujo KLB, Slattum P, Van Ness PH, Inouye SK. Benzodiazepine and opioid use and the duration of intensive care unit delirium in an older population. Crit Care Med. (2009) 37:17783. doi: 10.1097/CCM.0b013e318192fcf9

71. Wang J, Ji Y, Wang N, Chen W, Bao Y, Qin Q, et al. Risk factors for the incidence of delirium in cerebrovascular patients in a neurosurgery intensive care unit: a prospective study. J Clin Nurs. (2018) 27:40715. doi: 10.1111/jocn.13943

72. Akkurt I, Cetin C, Erdogan AM, Dincel GC, Ceylan AF, Kisa U, et al. Cerebral ischaemia/reperfusion injury could be managed by using tramadol. Neurol Res. (2018) 40:774-84. doi: 10.1080/01616412.2018. 1477556
73. Unchiti K, Leurcharusmee P, Samerchua A, Pipanmekaporn T, Chattipakorn $\mathrm{N}$, Chattipakorn SC. The potential role of dexmedetomidine on neuroprotection and its possible mechanisms: evidence from in vitro and in vivo studies. Eur J Neurosci. (2021) 54:7006-47. doi: 10.1111/ejn.15474

74. Siddiqui NT, Yousefzadeh A, Yousuf M, Kumar D, Choudhry FK, Friedman Z. The effect of gabapentin on delayed discharge from the postanesthesia care unit: a retrospective analysis. Pain Pract. (2018) 18:1822. doi: $10.1111 /$ papr. 12575

Conflict of Interest: The authors declare that the research was conducted in the absence of any commercial or financial relationships that could be construed as a potential conflict of interest.

Publisher's Note: All claims expressed in this article are solely those of the authors and do not necessarily represent those of their affiliated organizations, or those of the publisher, the editors and the reviewers. Any product that may be evaluated in this article, or claim that may be made by its manufacturer, is not guaranteed or endorsed by the publisher.

Copyright (c) 2022 Kvolik, Koruga and Skiljic. This is an open-access article distributed under the terms of the Creative Commons Attribution License (CC BY). The use, distribution or reproduction in other forums is permitted, provided the original author(s) and the copyright owner(s) are credited and that the original publication in this journal is cited, in accordance with accepted academic practice. No use, distribution or reproduction is permitted which does not comply with these terms. 